\title{
Polarization Independent Multiple Fano Resonances in Plasmonic \\ Nonamers for Multimode-Matching Enhanced Multiband \\ Second-Harmonic Generation
}

\section{Supporting Information}

Shao-Ding Liu, ${ }^{\dagger,+}$ Eunice Sok Ping Leong, ${ }^{\#}$ Guangcan Li, ${ }^{\perp}$ Yidong Hou, ${ }^{\perp}$ Jie Deng, ${ }^{\#}$ Jing Hua

Teng, ${ }^{\#}$ Hock Chun Ong, ${ }^{\S}$ and Dang Yuan Lei ${ }^{\perp, *}$

†Key Lab of Advanced Transducers and Intelligent Control System of Ministry of Education, Taiyuan University of Technology, Taiyuan 030024, P. R. China.

Department of Physics and Optoelectronics, Taiyuan University of Technology, Taiyuan 030024, P. R. China.

"Institute of Materials Research and Engineering, A*STAR, 2 Fusionopolis Way, Singapore 138634. ${ }^{\S}$ Department of Physics, The Chinese University of Hong Kong, Hong Kong, China.

${ }^{\perp}$ Department of Applied Physics, The Hong Kong Polytechnic University, Hong Kong, China.

*Address correspondence to dylei@polyu.edu.hk

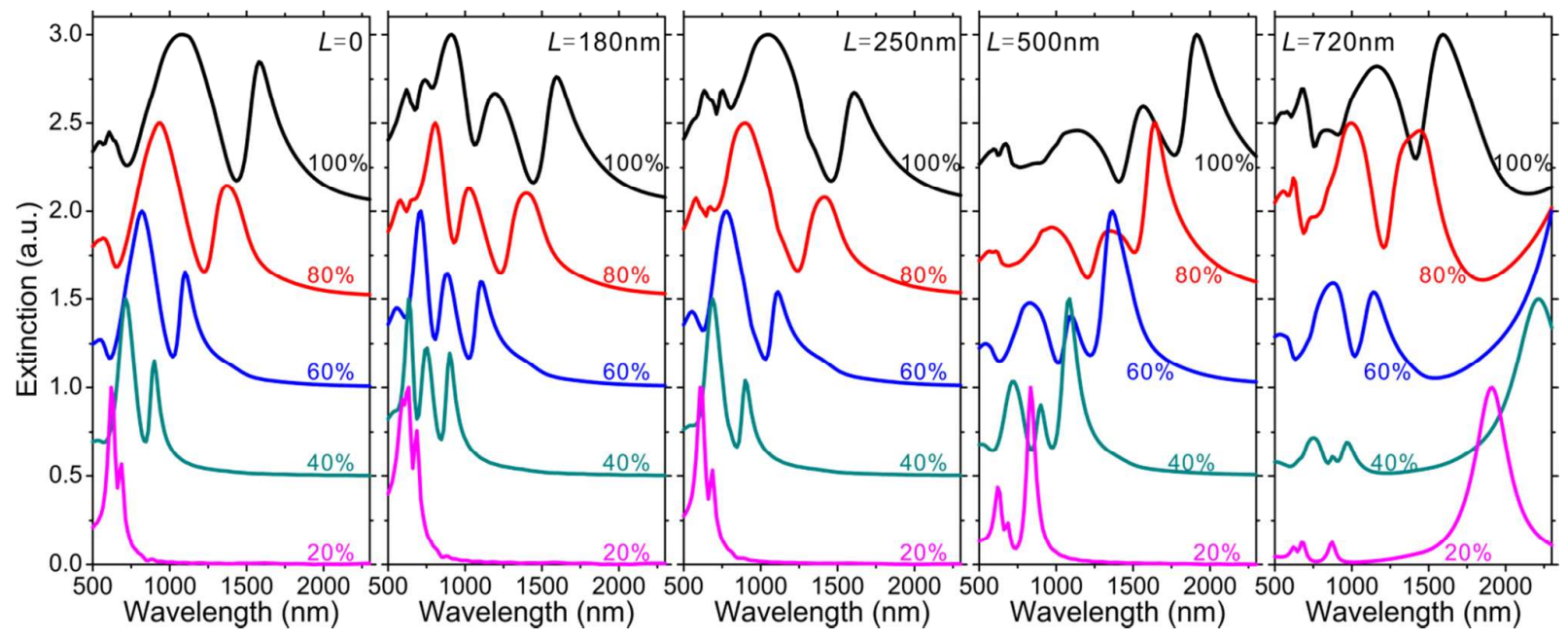

Figure S1. Extinction spectra of the nonamers shown in Figure 2 by scaling down their geometry parameters at different factors, where the thickness $(50 \mathrm{~nm})$ and the separation between adjacent 
layers $(30 \mathrm{~nm})$ are assumed to be constants, and the outer layer is supposed to be a square ring for each scaled nonamer with $L=720 \mathrm{~nm}$.

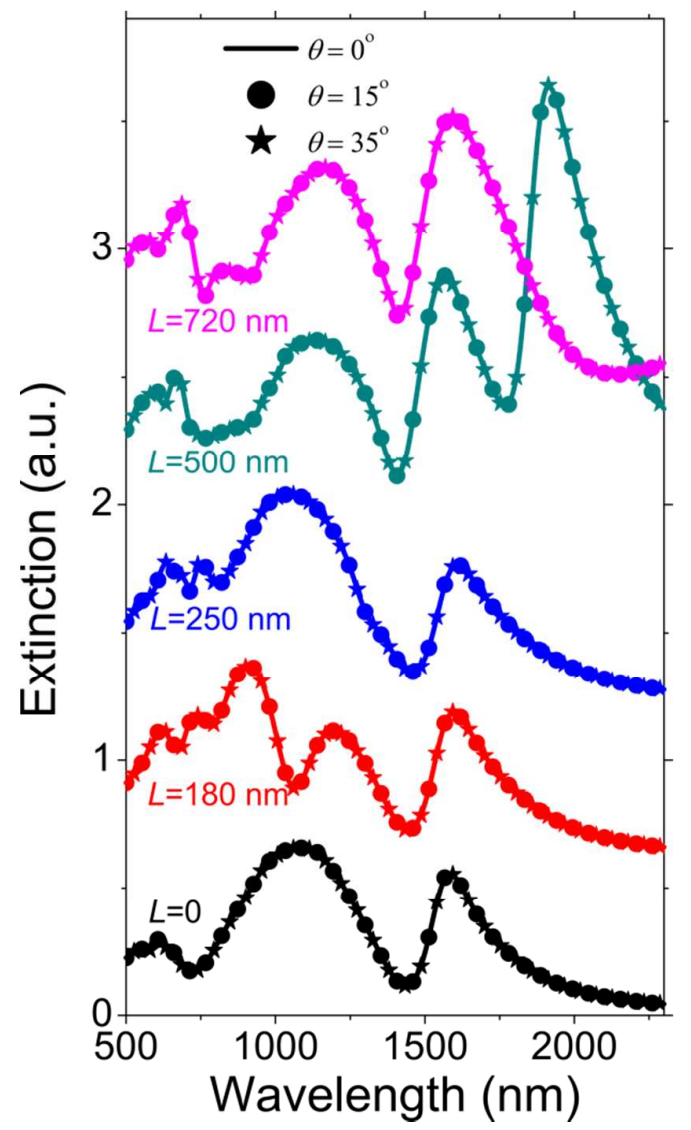

Figure S2. Extinction spectra of the nonamers shown in Figure 2 with one symmetric $\left(0^{\circ}\right.$, solid lines $)$ and two non-symmetric $\left(15^{\circ}\right.$ and $35^{\circ}$, circles and stars $)$ incident polarizations.

Lovera et al. have demonstrated that for the Fano resonances generated in a nanorod quadrumer, many remarkable properties like resonance crossing/anti-crossing behaviors have been observed by manipulating geometry parameters. ${ }^{28}$ An extended coupled oscillator (ECO) model has been developed to provide physical insights into these interesting properties of the system. It is shown that the resonance crossing/anti-crossing behaviors can be understood due to the variation of the ECO parameters such as coupling coefficient between the two oscillators. The same collective bright and dark modes are involved in the formation of the Fano resonances. However, for the nonamers considered in our study, the near-field distributions shown in Figure 4 indicate that the corresponding subradiant modes of the four Fano resonances marked with dashed lines in Figure 3 are in fact 
different hybridized collective resonances.

In order to better illustrate these hybridized collective modes, two plasmon hybridization schemes of the nonamer are presented in Figure S3, where only the longitudinal dipole resonance of individual nanoparticles are considered. Figure S3a shows plasmon hybridization between the antibonding dipole mode of the surrounding dimers and the dipole mode of the nanocross, where the hybridized antibonding mode of the nonamer (white solid energy level) is the corresponding superradiant mode of the four Fano resonances (Figure 4a and 4c), and the hybridized bonding mode (green solid energy level) is the corresponding subradiant mode of the second Fano resonance (Figure 4b, 4c and 4g). Figure S3b shows the plasmon hybridization between the bonding dipole mode of the surrounding dimers and the dipole mode of the nanocross, where the resulting hybridized antibonding mode of the nonamer (light blue solid energy level) is the corresponding subradiant mode of the first Fano resonance (Figure 4d). The hybridized bonding mode (yellow dashed energy level) is similar to that of the subradiant mode of the third Fano resonance (Figure $4 \mathrm{~h}$ and $4 \mathrm{j}$ ), but it should be noted that the dipole moment of the outer nanorods are induced through near-field coupling as we have shown in the main text. This is different from that of a plasmonic dipolar resonance because the resonance energy of the third Fano resonance is almost unchanged with variation of the length of the outer nanorods. As for the subradiant mode of the fourth Fano resonance (Figure 4i), the higher-order mode of the inner nanorods are involved in the plasmon hybridization, and one cannot get the corresponding collective mode by considering only the dipolar resonance of individual nanoparticles. Therefore, it is concluded that the four sets of the Fano resonances shown in Figure 3 possess different subradiant modes and thus different formation mechanisms. 
Nevertheless, in the following we will show that the ECO model can be used to describe the multiple Fano resonances generated in the nonamers. The inset of Figure S4 shows schematically three coupled resonators, which represent the three layers of the nonamers, respectively. Each resonator is driving by an external force for the ECO model, but it should be noted that the plasmons of the outer nanorods for the third Fano resonance and the plasmons of the inner nanorods for the fourth Fano resonance are in fact cannot be directly excited in the individual layers. The equations of motion can be written as,

$$
\begin{aligned}
& \ddot{x}_{1}+\gamma_{1} \dot{x}_{1}+\omega_{1}^{2} x_{1}+g_{12} x_{2}+g_{13} x_{3}=0.5 \dddot{P}_{t o t}+\alpha_{1} E_{e x t} \\
& \ddot{x}_{2}+\gamma_{2} \dot{x}_{2}+\omega_{2}^{2} x_{2}+g_{12} x_{1}+g_{23} x_{3}=0.5 \dddot{P}_{t o t}+\alpha_{2} E_{e x t} \\
& \ddot{x}_{3}+\gamma_{3} \dot{x}_{3}+\omega_{3}^{2} x_{3}+g_{13} x_{1}+g_{23} x_{2}=0.5 \dddot{P}_{t o t}+\alpha_{3} E_{e x t}
\end{aligned}
$$

where $g_{12}, g_{13}$ and $g_{23}$ are the coupling coefficients, the total dipole moment of the system $P_{\text {tot }}=\alpha_{1} x_{1}$ $+\alpha_{2} x_{2}+\alpha_{3} x_{3}, x_{1,2,3}$ are the amplitudes of the resonators, $\alpha_{1,2,3}, \omega_{1,2,3}$ and $\gamma_{1,2,3}$ are the corresponding polarizabilities, resonant frequencies and nonradiative damping, respectively. Suppose $E_{\mathrm{ext}}=E_{0} e^{\mathrm{i} \omega t}$, that is, an incident harmonic field, the displacements of the resonators can be written as $x_{1,2,3}(\omega)=$ $C_{1,2,3}(\omega) e^{\mathrm{i} \omega t}$, where $C_{1,2,3}$ are the oscillation amplitudes. The squared modulus of the sum of the amplitudes, $\left|C_{1}+C_{2}+C_{3}\right|^{2}$, can then be used to model the scattering spectrum. Figure $\mathrm{S} 4$ shows the fits with the ECO model, where the parameters used are presented in Table S1, and the theoretical fits of the ECO model agree well with the calculated scattering spectra by FDTD. 

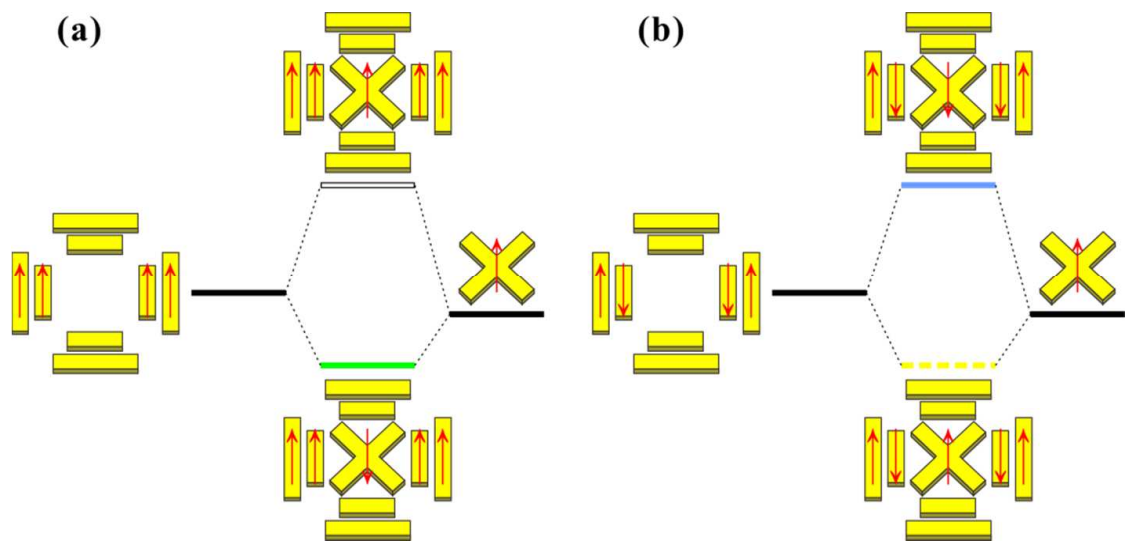

Figure S3. Plasmon hybridization schemes of the nonamer, where only the dipole modes of individual nanorods and nanocross are considered. (a) Plasmon hybridization between the antibonding dipole mode of the surrounding dimers and the dipole mode of the nanocross. (b) Plasmon hybridization between the bonding dipole mode of the surrounding dimers and the dipole mode of the nanocross.

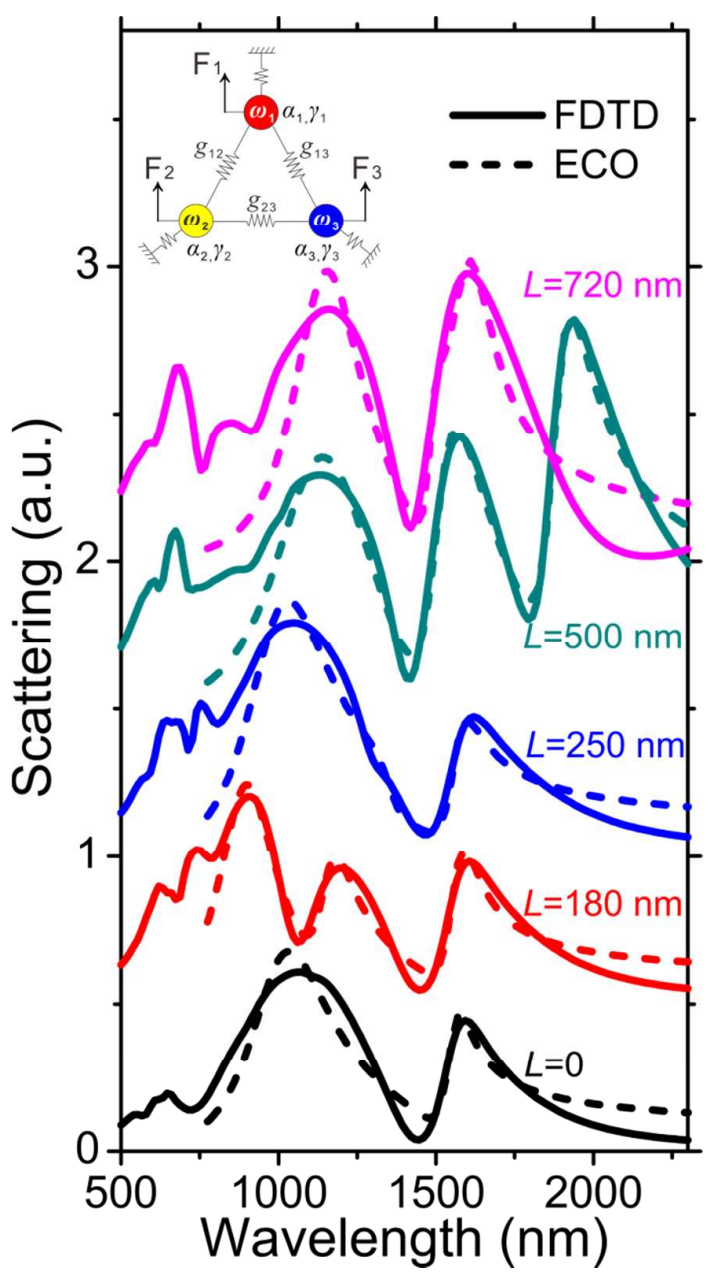

Figure S4. Comparison between calculated scattering spectra with FDTD (solid lines) and with the ECO model (dashed lines), where the inset shows a schematic view of the ECO model. 
Table S1. Parameters for the ECO model for varying nanorod length $L$ of the outer ring layer

\begin{tabular}{cccccccccccccc}
\hline \hline$L[\mathrm{~nm}]$ & $\omega_{1}[\mathrm{eV}]$ & $\omega_{2}[\mathrm{eV}]$ & $\omega_{3}[\mathrm{eV}]$ & $g_{12}\left[\mathrm{eV}^{2}\right]$ & $g_{13}\left[\mathrm{eV}^{2}\right]$ & $g_{23}\left[\mathrm{eV}^{2}\right]$ & $E_{0}$ & $\gamma_{1}[\mathrm{eV}]$ & $\gamma_{2}[\mathrm{eV}]$ & $\gamma_{3}[\mathrm{eV}]$ & $\alpha_{1}\left[\mathrm{eV}^{-1}\right]$ & $\alpha_{2}\left[\mathrm{eV}^{-1}\right]$ & $\alpha_{3}\left[\mathrm{eV}^{-1}\right]$ \\
\hline 0 & 0.859 & 1.223 & - & 0.289 & - & - & 1.263 & 0.031 & 0.174 & - & 0.101 & 0.241 & - \\
180 & 0.916 & 1.112 & 1.316 & 0.323 & 0.338 & 0.274 & 1.746 & 0.039 & 0.090 & 0.148 & 0.101 & 0.100 & 0.102 \\
250 & 0.890 & 1.080 & 1.080 & 0.156 & 0.288 & 0.383 & 1.150 & 0.030 & 0.104 & 0.104 & 0.100 & 0.216 & 0.142 \\
500 & 0.800 & 0.904 & 1.028 & 0.192 & 0.349 & 0.235 & 0.801 & 0.031 & 0.067 & 0.102 & 0.269 & 0.344 & 0.180 \\
720 & 0.835 & 0.912 & 0.997 & 0.044 & 0.140 & 0.255 & 1.068 & 0.030 & 0.031 & 0.159 & 0.100 & 0.100 & 0.115 \\
\hline \hline
\end{tabular}

The mode-matching condition, that is, the formation of amplified nonlinear excitation source and the increased scattering efficiency at the emission wavelength, is critical for enhanced SHG. In the main text, we have shown that the nonlinear sources are enhanced at several spectral positions using the multiple Fano resonances generated in the nonamers, and the scattering efficiencies of SH emissions can concurrently be increased by several higher-order resonances in the visible region. Therefore, the multimode-matching condition can be achieved with these rationally-designed nonamer clusters, leading to production of the strong SH emissions at multiple wavelength bands simultaneously. To better illustrate the enhanced multiband SH emissions in the structures, the near-field distribution at the $\mathrm{FH}$ and corresponding $\mathrm{SH}$ wavelengths as well as the angular distribution of the SH emission intensity are presented in Figures S5, S6, and S7, respectively.

For the two nonamer clusters with $L=0$ and $180 \mathrm{~nm}$, strong near-field enhancement can be seen for the excitation of the first and the second Fano resonances (upper two panels, Figure S5), and the amplification of nonlinear sources dominates the SHG efficiency, leading to relatively strong SH emissions at these spectral positions (upper two panels, Figure S6 and S7). In addition, several higher-order resonance modes marked with the magenta bars appear within the harmonic spectral ranges (black dashed lines, Figure $7 \mathrm{a}$ and $7 \mathrm{~b}$ ), which can be excited by the nonlinear sources and be used to amplify the SH emissions. When the FH wavelengths are away from the Fano resonances, 
the nonlinear excitation sources become weaker but the strong scattering at the SH wavelengths plays a dominant role in the SH far-field response (rather than in the near-field regime), resulting in even stronger SH emissions from the nonamers with $L=0$ and $180 \mathrm{~nm}$ (lower two panels, Figure S6 and S7). The importance of an increased scattering efficiency for SHG is further confirmed by comparing the results for the nonamers with $L=180$ and 250. The latter structure has a higher-order resonance at the $\mathrm{SH}$ emission position of its first Fano resonance (FH wavelength), fulfilling the mode-matching condition (light blue bar, Figure 7c). Although the FH near-field enhancement for the latter structure is much smaller than that for the former structure (upper panel, Figure S5), the SH emission intensity for the former can be 5 times stronger (upper panel, Figure S6 and S7).

By enlarging $L$ to $500 \mathrm{~nm}$, the FH near-field enhancement factors for the third and fourth Fano resonances are much larger than the nonamers with $L=0,180$ and $250 \mathrm{~nm}$ due to stronger plasmonic coupling between adjacent nanoparticles (middle two panels, Figure S5), which further increases the strength of the nonlinear excitation sources. Additionally, the higher-order resonances at 700 and $1007.5 \mathrm{~nm}$ (lower two panels, Figure S5) for this structure are very close to the SH wavelengths of the FH Fano resonances and their near-field enhancement factors are comparable, leading to the realization of a multimode-matching condition. As a result, the $\mathrm{SH}$ emission intensities of the nonamers with $L=500$ and $720 \mathrm{~nm}$ are significantly increased at multiple wavelength bands due to coherent amplification of the nonlinear excitation sources by the Fano resonances and efficient scattering enhanced outcoupling by the higher-order modes (lower four panels, Figures S6 and S7). 

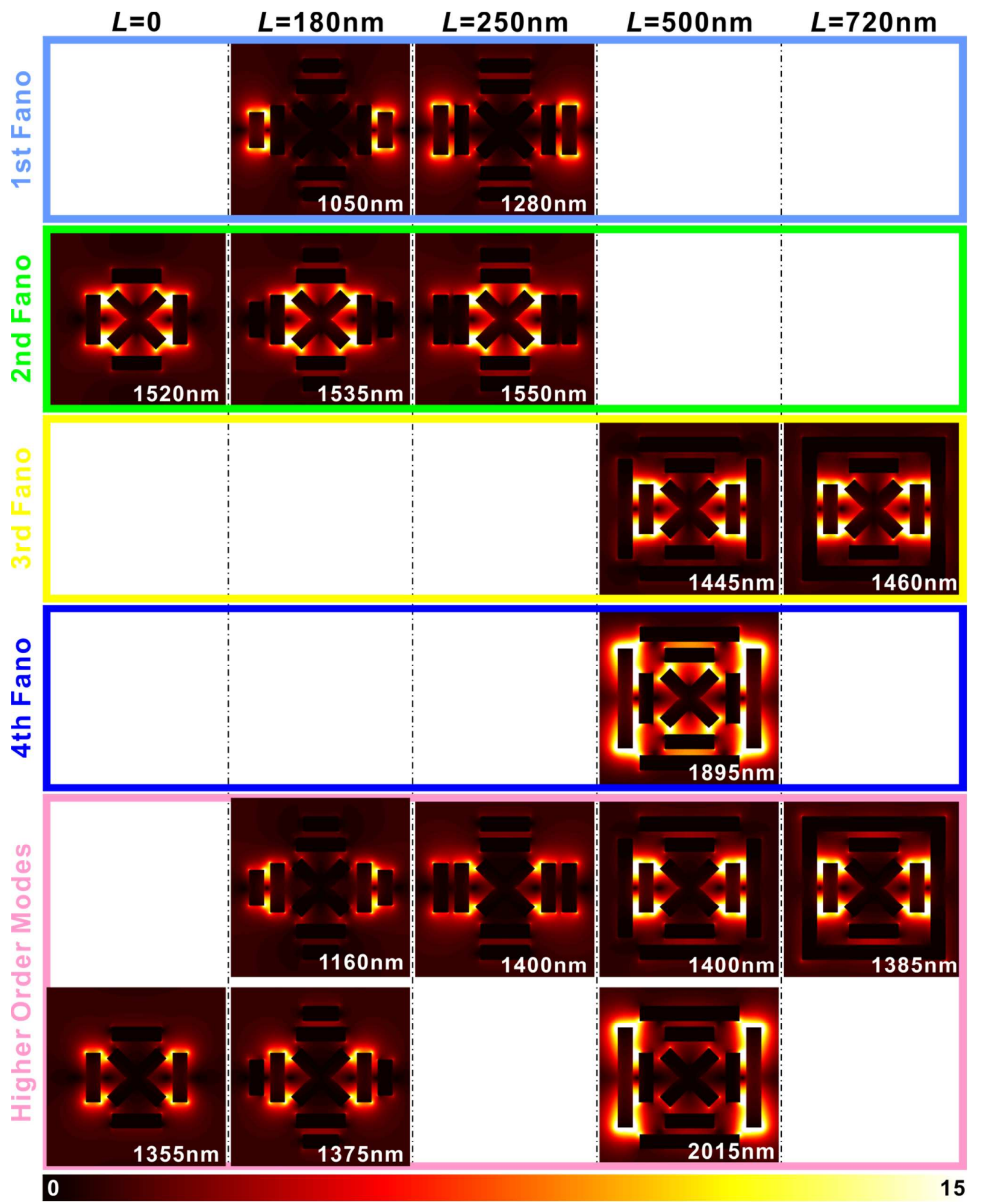

Figure S5. Normalized FH near-field enhancement distributions in the plasmonic nonamers at the $\mathrm{SH}$ emission peaks marked with the color bars in Figure 7 of the main text. 

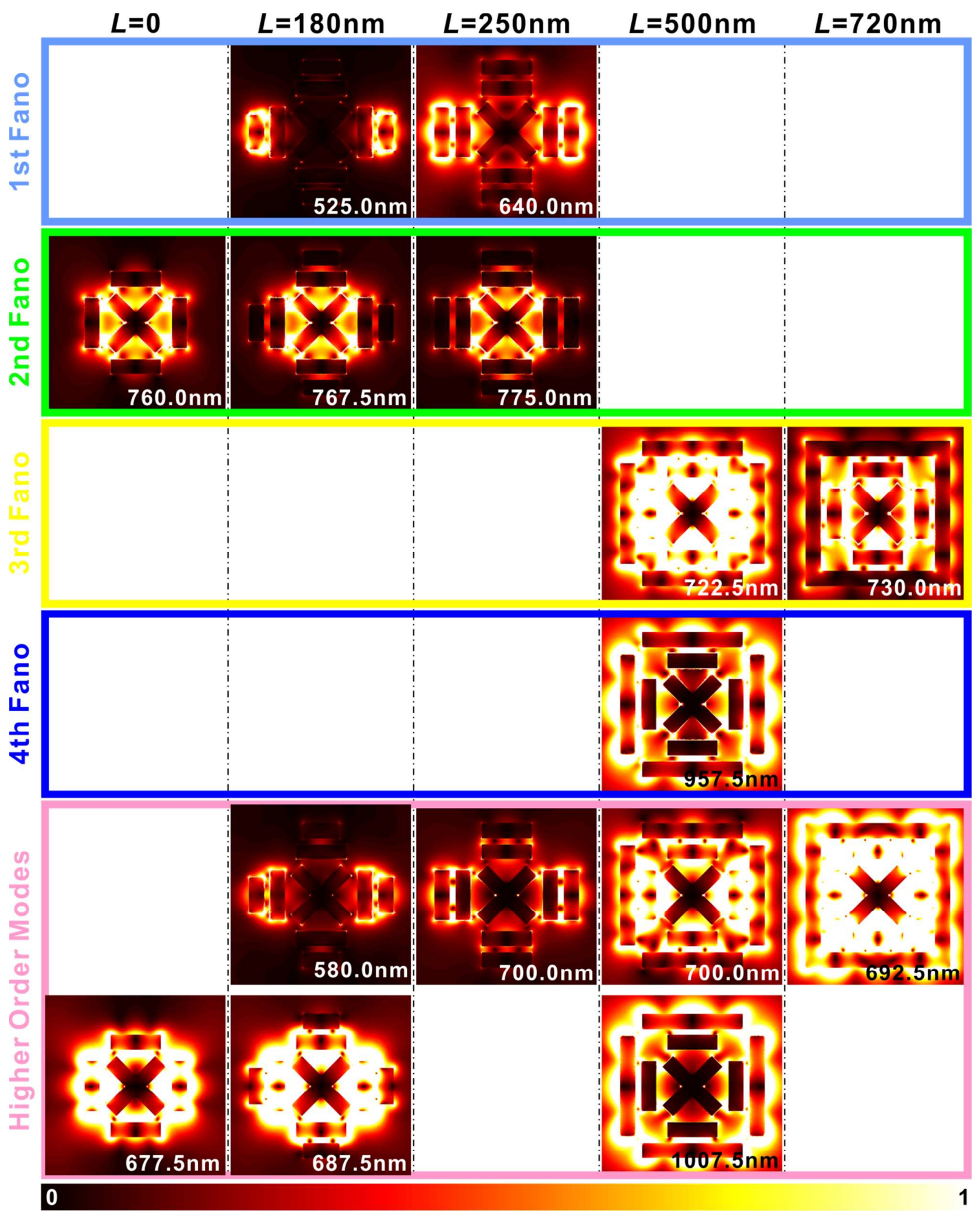

Figure S6. Normalized SH near-field distributions in the plasmonic nonamers at the SH emission peaks marked with the color bars in Figure 7 of the main text. 

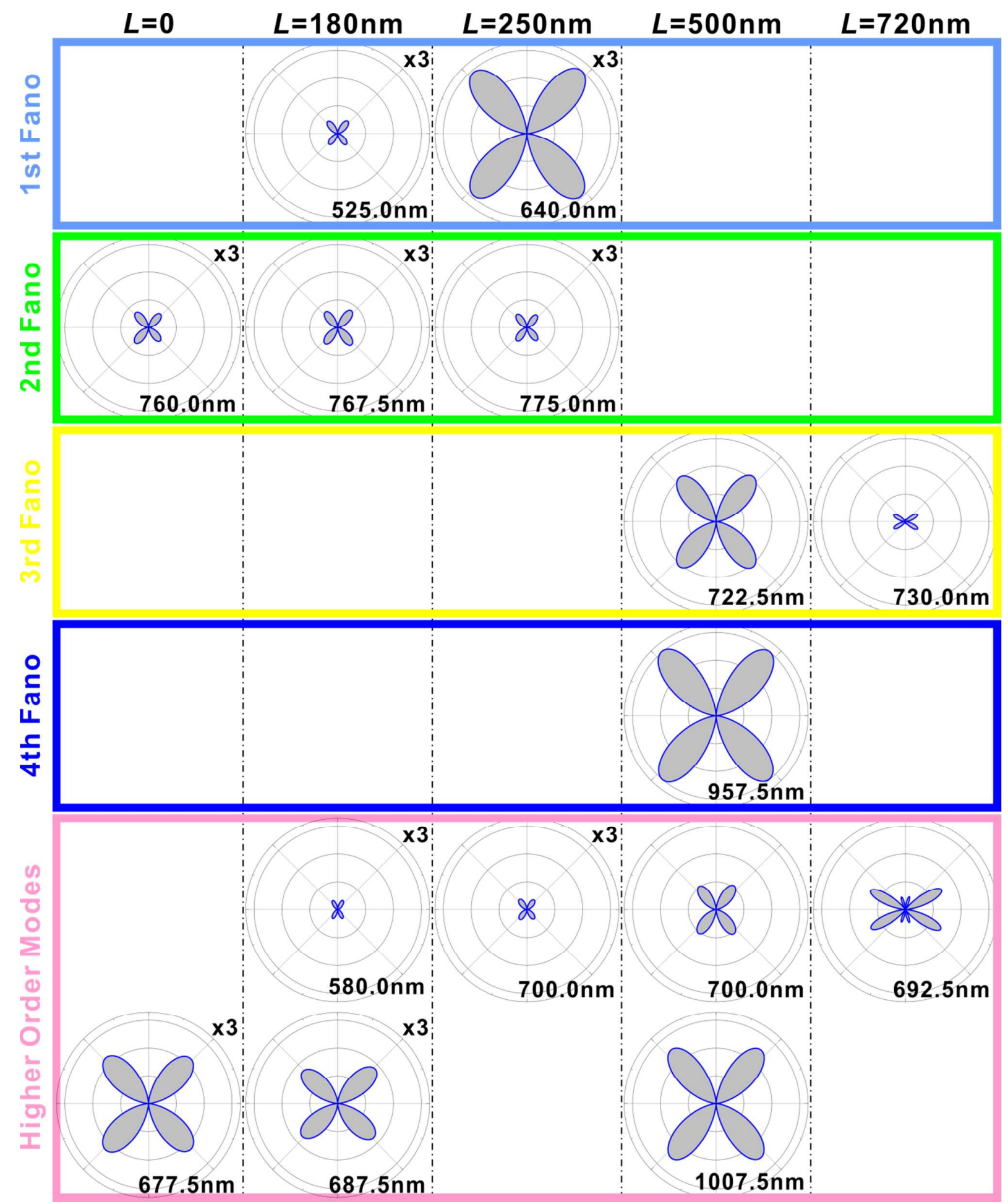

Figure S7. Angular plots of the far-field intensities in the $y-\mathrm{z}$ plane for the SH emission peaks marked with the color bars in Figure 7 of the main text, where the intensities are scaled by a factor of 3 for $L=0,180$ and $250 \mathrm{~nm}$. 

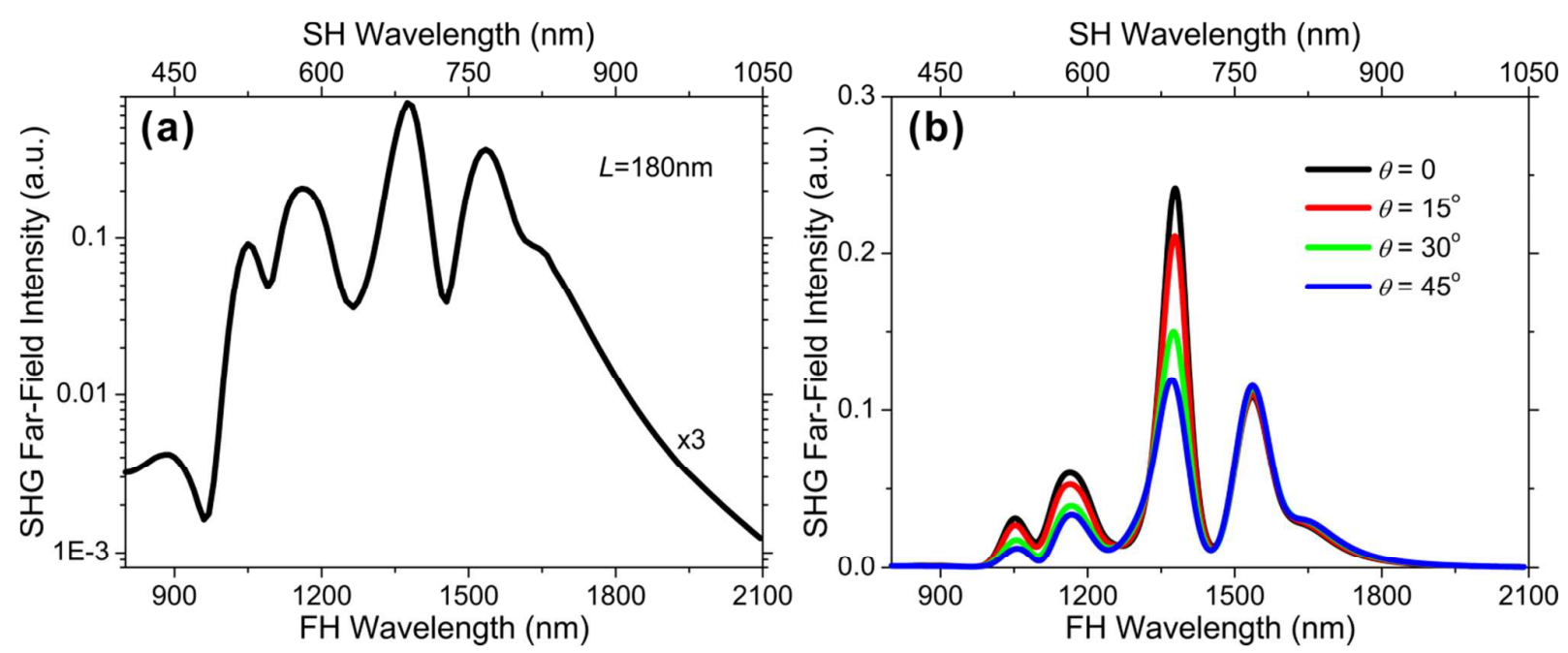

Figure S8. Calculated wavelength-dependent SHG far-field intensity in logarithmic scale for the nonamer with $L=180 \mathrm{~nm}$ (a) and its incidence polarization dependence (b).
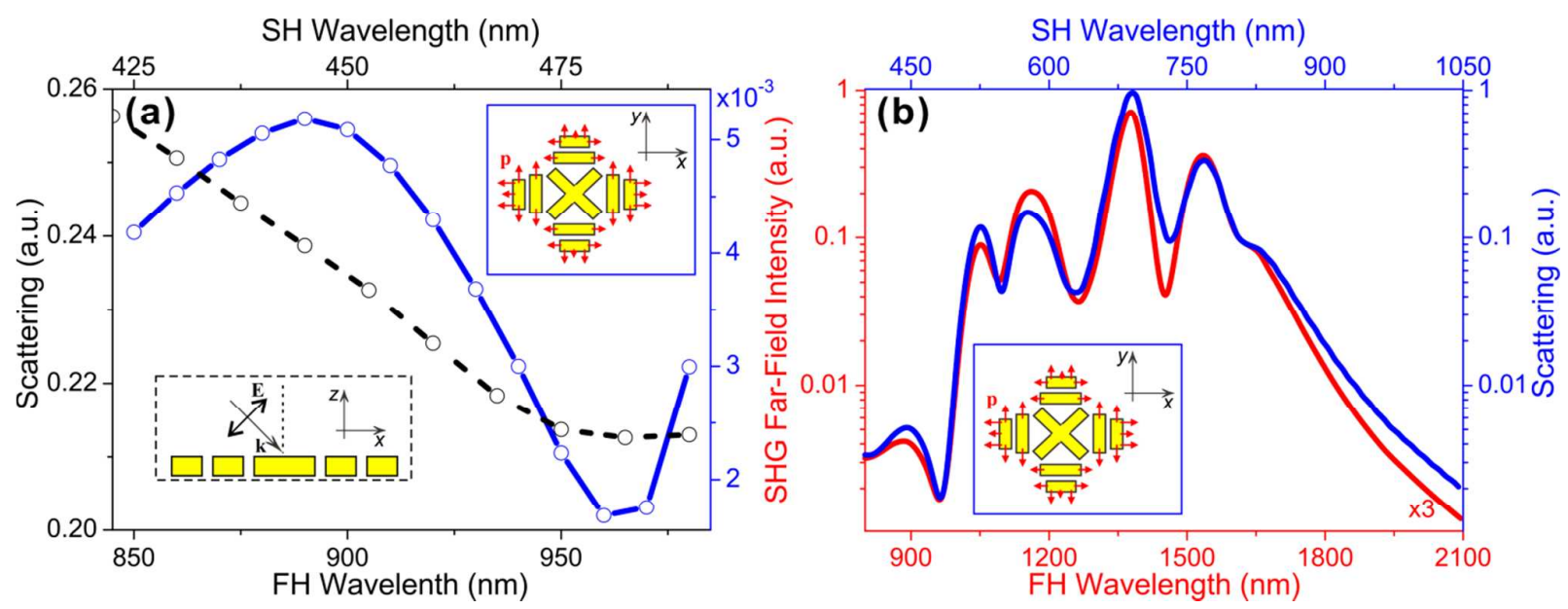

Figure S9. (a) Black dashed line: scattering spectrum calculated with an obliquely incident plane wave in the $\mathrm{SH}$ wavelength range $\left(45^{\circ}\right)$; Blue solid line: scattering spectrum calculated with $\mathrm{SH}$ nonlinear sources. (b) Comparison between the far-field SH emission intensity (red solid line) and the scattering spectrum in the SH wavelength range calculated with nonlinear sources (blue solid line).

The SHG emitted by a gold nanorod dimer is studied to further confirm the effect of the enhanced scattering and nonlinear source, ${ }^{78}$ and it is also used to quantify the SHG emission intensity of the designed nonamers. In addition to the SHG emission peak observed with $1575 \mathrm{~nm}$ incidence, where the nonlinear sources are enhanced by the bonding dipole mode of the dimer (the 
green bar and Figure S10e), an even stronger SHG emission peak is observed with $1365 \mathrm{~nm}$ incidence (the magenta bar and Figure S10d). Under oblique incident excitation, there is a resonance shoulder in the scattering spectrum around $685 \mathrm{~nm}$ (black dashed line, the first panel of Figure S10a). This resonance mode is more effectively excited with an electric dipole or the nonlinear sources (red and blue solid lines, the first panel of Figure S10a). The near-field profile shown in Figure S10b indicates that the resonance at $685 \mathrm{~nm}$ is an antibonding quadrupole mode of the dimer, which has the same near-field distribution as that of the SH emission (Figure S10f and S10g). ${ }^{78}$ Therefore, the stronger SHG emission with $1365 \mathrm{~nm}$ incidence is related to excitation of the antibonding quadrupole mode in the $\mathrm{SH}$ wavelength range, and the enhanced scattering efficiency leads to the stronger SH emission. This observation further confirms that the low intensity scattering of the nonamer under an oblique incidence at $440 \mathrm{~nm}$ is caused by the relatively weak coupling of the incident field with higher-order resonances (Figure S9a), and an efficient scattering enhanced outcoupling with plasmon resonance is therefore important for efficient SHG. The scattering spectra shown in the first panel of Figure S10a also reveal that the bonding quadrupole mode of the dimer at around $760 \mathrm{~nm}$ can be excited under oblique incidence or with the electric dipole (Figure S10c), but it cannot be coupled with the nonlinear sources. 


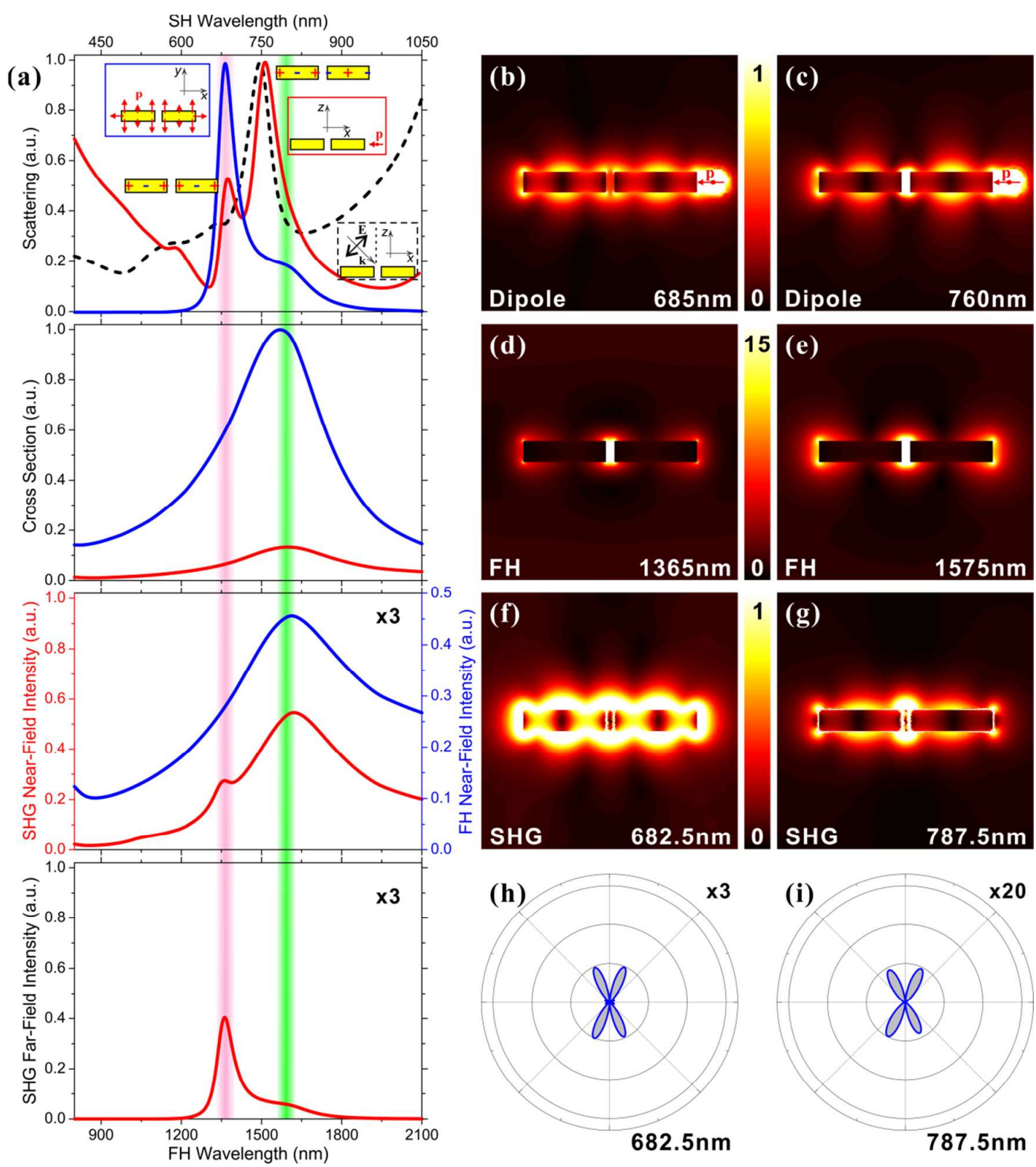

Figure S10. (a) Simulated scattering and SH spectra of a gold nanorod dimer. The first panel shows the simulated scattering spectra in the SH wavelength range with three excitation methods, Black dashed line: under an oblique incident $\left(45^{\circ}\right)$; Red solid line: excited by an electric dipole; Blue solid line: excited with the SH nonlinear sources. The second panel shows the extinction (blue solid line) and absorption (red solid line) spectra in the FH wavelength range. The third panel shows the variation of the SH (red line) and FH (blue line) near-field intensity against FH wavelength and the fourth panel shows the variation of the SH far-field intensity. Each nanorod has a length of $290 \mathrm{~nm}$, a width of $75 \mathrm{~nm}$ and a thickness of $50 \mathrm{~nm}$. The gap separation of the two nanorods is $30 \mathrm{~nm}$. The SH near- and far-field intensities are normalized with that of the designed nanoclusters shown in Figure 7, and the intensities are scaled by a factor of 3. (b \& c) Near-field profiles of the antibonding (b) and bonding (c) quadrupole modes excited by an electric dipole, respectively. (d \& e) FH near-field enhancement distributions at $1365 \mathrm{~nm}$ (d) and $1575 \mathrm{~nm}$ (e). (f - i) SH near-field distributions at $682.5 \mathrm{~nm}(\mathrm{f})$ and $787.5 \mathrm{~nm}(\mathrm{~g})$ and corresponding angular intensity emission plots (h $\&$ i), where the far-field intensities in (h) and (i) are scaled by a factor of 3 and 20, respectively. 